\title{
BMJ Open Protocol of a prospective, multicentre phase I study to evaluate the safety, tolerability and preliminary efficacy of the bispecific PSMAxCD3 antibody CC-1 in patients with castration- resistant prostate carcinoma
}

Jonas S Heitmann (D) ,1,2 Juliane S Walz,, ${ }^{1,2}$ Martin Pflügler, ${ }^{1,3}$ Joseph Kauer, $^{3}$ Richard F Schlenk, ${ }^{4,5,6}$ Gundram Jung, ${ }^{3}$ Helmut R Salih ${ }^{1,2}$

To cite: Heitmann JS, Walz JS Pflügler M, et al. Protocol of a prospective, multicentre phase I study to evaluate the safety, tolerability and preliminary efficacy of the bispecific PSMAxCD3 antibody CC-1 in patients with castration-resistant prostate carcinoma. BMJ Open 2020;10:e039639. doi:10.1136/ bmjopen-2020-039639

- Prepublication history and additional materials for this paper is available online. To view these files, please visit the journal online (http://dx.doi. org/10.1136/bmjopen-2020039639).

RFS, GJ and HRS contributed equally.

Received 21 April 2020 Revised 20 August 2020 Accepted 24 August 2020

D) Check for updates

(c) Author(s) (or their employer(s)) 2020. Re-use permitted under CC BY-NC. No commercial re-use. See rights and permissions. Published by BMJ.

For numbered affiliations see end of article.

Correspondence to Professor Helmut R Salih; helmut.salih@med.unituebingen.de

\section{ABSTRACT}

Introduction Prostate cancer is the second most common cancer in men worldwide. When the disease becomes resistant to androgen-deprivation therapy, treatment options are sparse. To address the high medical need in castration-resistant prostate cancer (CRPC), we generated a novel PSMAxCD3 bispecific antibody termed CC-1. CC-1 binds to prostate-specific membrane antigen that is expressed on prostate cancer cells and tumour vessels, thereby allowing a dual anticancer effect.

Methods and analysis This first in human clinical study is a prospective and multicentre trial which enrols patients with metastatic CRPC after failure of established third-line therapy. CC-1 is applied after prophylactic interleukin-6 receptor blockade with tocilizumab (once $8 \mathrm{mg} / \mathrm{kg}$ body weight). Each patient receives at least one cycle of CC-1 over a time course of 7 days in an inpatient setting. If clinical benefit is observed, up to five additional cycles of CC-1 can be applied. The study is divided in two parts: (1) a dose escalation phase with intraindividual dose increase from $28 \mu \mathrm{g}$ to the target dose of $1156 \mu \mathrm{g}$ based on a modified fast titration design by Simon et a/ to determine safety, tolerability and the maximum tolerated dose (MTD) as primary endpoints and (2) a dose expansion phase with additional 14 patients on the MTD level of part (1) to identify first signs of efficacy. Secondary endpoints compromise overall safety, tumour response, survival and a translational research programme with, among others, the analysis of CC-1 half-life, the induced immune response, as well as the molecular profiling in liquid biopsies.

Ethics and dissemination The PSMAXCD3 study was approved by the Ethics Committee of The University Hospital Tübingen (100/2019AMG1) and the Paul-EhrlichInstitut (3684/02). Clinical trial results will be published in peer-reviewed journals.

Trial registration numbers ClinicalTrials.gov Registry (NCT04104607) and ClinicalTrials.eu Registry (EudraCT2019-000238-20).

\section{INTRODUCTION}

Prostate cancer is the second most common cancer in men worldwide, with
Strengths and limitations of this study

- This first in human (FIH) study not only evaluates the clinical safety and maximum tolerated dose of a novel PSMAXCD3 antibody (CC-1) in prostate cancer, but will also unravel first signs of efficacy in a dose expansion cohort

- The ethical dilemma for patients treated at early time points during dose escalation in FIH studies is addressed by rapid intrapatient dose escalation.

- The novel IgG-based format of CC-1 not only prolongs serum half-life but also reduces off-target $T$ cell activation which, together with pre-emptive interleukin-6 receptor blockade using tocilizum$a b$, results in fewer side effects and in turn allows for application of truly effective bispecific antibody doses.

- Close monitoring by Data Safety Monitoring Board to protect patients' interests and study safety.

- Development and first clinical application of the drug is exclusively financed by public resources.

estimated 1100000 cases and 307000 deaths in 2012. ${ }^{2}$ Androgen-deprivation therapy is standard of care first-line therapy of advanced prostate cancer. However, frequently prostate carcinoma develops resistance to first-line therapy. Notably, most drugs established for treatment of these castration-resistant prostate carcinomas (CRPCs) (eg, abiraterone acetate, enzalutamide) still act on the androgen axis. Applied prior to or after treatment with chemotherapeutic agents (eg, docetaxel, cabazitaxel), these therapies slow down disease progression and improve survival to a moderate extent. Abiraterone resulted in a median overall survival (OS) benefit of 4.6 months post-docetaxel and of 4.4 months in 
chemotherapy-naive patients. ${ }^{34}$ Enzalutamide resulted in a median OS benefit of 4.8 months post-chemotherapy. ${ }^{5}$ The chemotherapeutic agents docetaxel and cabazitaxel resulted in a median OS benefit of 2.4 months. ${ }^{67}$ In case of progression/relapse, for example, abiraterone can be used after enzalutamid or after docetaxel and vice versa. Importantly, the best sequence of treatments has not been finally established, and any drug employed after the third line of treatment is associated with only limited clinical benefit.

Novel strategies have to be developed to address the medical need of this patient population. Of particular interest in this context are strategies to target the prostate-specific membrane antigen (PSMA), which is expressed, at least to some extent, in almost all patients (up to 98\%) with a highly tumour-restricted expression pattern. Targeted radiotherapy approaches using for example, Lutetium-177-PSMA ${ }^{8}$ showed efficacy and a tolerable toxicity profile on treatment of patients with metastatic disease. However, the duration of achieved responses is limited, and many patients do not at all benefit from this treatment option. Meanwhile, immunotherapy has become a mainstay of oncological treatment. Available strategies comprise immune checkpoint blocking antibodies (eg, nivolumab, pembrolizumab) that are approved for treatment of various solid tumours including non-small cell lung cancer, melanoma and renal cell carcinoma. ${ }^{9-11}$ However, these checkpoint inhibitors have shown only limited efficacy in prostate cancer. ${ }^{12}$ Other successful antibody-based strategies that mobilise $\mathrm{T}$ cells against cancer comprise bispecific antibodies (bsAbs) and chimeric antigen receptor T (CART) cells. The first stimulate the T cell receptor/CD3-complex with their effector part after binding their target antigen on tumour cells. The latter are functionally closely related to the bsAb, as CART cells can be considered as genetically modified T cells with an integrated bsAb (CD3 signalling unit anchored in the T cell). ${ }^{13}$ The most advanced reagent in the class of bsAbs is the CD19xCD3 bsAb blinatumomab (Amgen) approved for treatment of acute lymphoblastic leukaemia in the bispecific single-chain (BiTE) format. ${ }^{1415}$ Like bsAb, CART cells are mainly established for treatment of lymphoid malignancies, ${ }^{16}$ and so far both, bsAbs and CART cells, are less effective if applied against solid tumours compared with haematological malignancies. ${ }^{17}$ In our view, a major problem is the lacking accessibility of solid tumours for the effector cells, be it for CART cells or bsAb-stimulated T cells. ${ }^{18}{ }^{19}$ Sustained therapeutic success of both, bsAb and CART cells are further limited by the severe side effects, in particular the potentially lethal cytokine-release syndrome (CRS) as most important group toxicity. At present, if CRS occurs, it is treated with interleukin-6 receptor (IL-6R) blockade using tocilizumab. ${ }^{20}$

We have developed an optimised bsAb with PSMAxCD3 specificity (CC-1) that, on application after pre-emptive IL-6R blockade holds promise to overcome the above described limitations. Of note, targeting PSMA with bsAbs not only holds promise to potentially induce more pronounced 'immediate effects' compared with PSMAtargeted radiotherapy, but may also stimulate immunological memory and thus mediate long-term efficacy. ${ }^{21}$ In our first in human (FIH) study reported here, we evaluate CC-1 in patients with metastatic CRPC to determine overall safety and tolerability, as well as the maximum tolerated dose (MTD) and first signs of efficacy.

\section{METHODS AND ANALYSIS}

\section{Properties of the bsAb CC- 1}

The bispecific PSMAxCD3 antibody CC-1 is an optimised IgG-like molecule (IgGsc format) with substantially improved serum half-life, especially when compared with the prototypical BiTE bsAb format. Specific modifications introduced in this proprietary format (disclosed patent application WO 2017/121905) ${ }^{22}$ further reduce aggregation tendency and thus unspecific 'off-target' $\mathrm{T}$ cell activation and immunogenicity. Its target is, in prostate carcinoma, expressed on both, tumour cells and tumour vessels. Vascular expression is expected to facilitate access of immune effector cells to the tumour site. Notably, CC-1 binds a unique PSMA epitope which allows for such dual targeting not only in prostate carcinoma, but also in squamous cell carcinoma of the lung (data provided in the patent application).

\section{Study design}

This multicentre, open-label, FIH phase I dose escalation and dose expansion study is designed to gain evidence of the MTD of CC-1 on prophylactic IL6-R blockade. Moreover, with the dose expansion part we aim to achieve first evidence of efficacy with the MTD of CC-1 in adult patients with CRPC after third-line therapy for a subsequent phase II study. The study is entitled 'First in human study to evaluate the safety, tolerability and preliminary efficacy of the bispecific PSMAxCD3 antibody CC-1 in patients with castration-resistant prostate carcinoma' (short title: 'DKTK_PMO_1605') and will be conducted within the framework of the German Cancer Consortium (DKTK).

\section{Study approach}

This FIH study consists of two parts (figure 1): (1) a dose escalation part (patients $n=10-72$ ) comprising a dose escalation with prophylactic IL-6R blockade $(8 \mathrm{mg} / \mathrm{kg}$ (body weight) tocilizumab) with intraindividual patient dose escalation (figure 2A-D) on CC-1-induced dose-limiting toxicity followed thereafter by a standard $3+3$ dose escalation design without intra-individual dose escalation (figure 3). In case of no observed dose-limiting toxicity, the maximal dose is achieved after intrapatient and interpatient dose escalation after four patients (figure 2A-D). The dose escalation part is followed by (2) a dose expansion part with an additional cohort $(n=14)$ receiving the defined MTD-level after pre-emptive tocilizumab application to refine/confirm the recommended phase II dose and to receive first signs of clinical efficacy (figure 2D). 


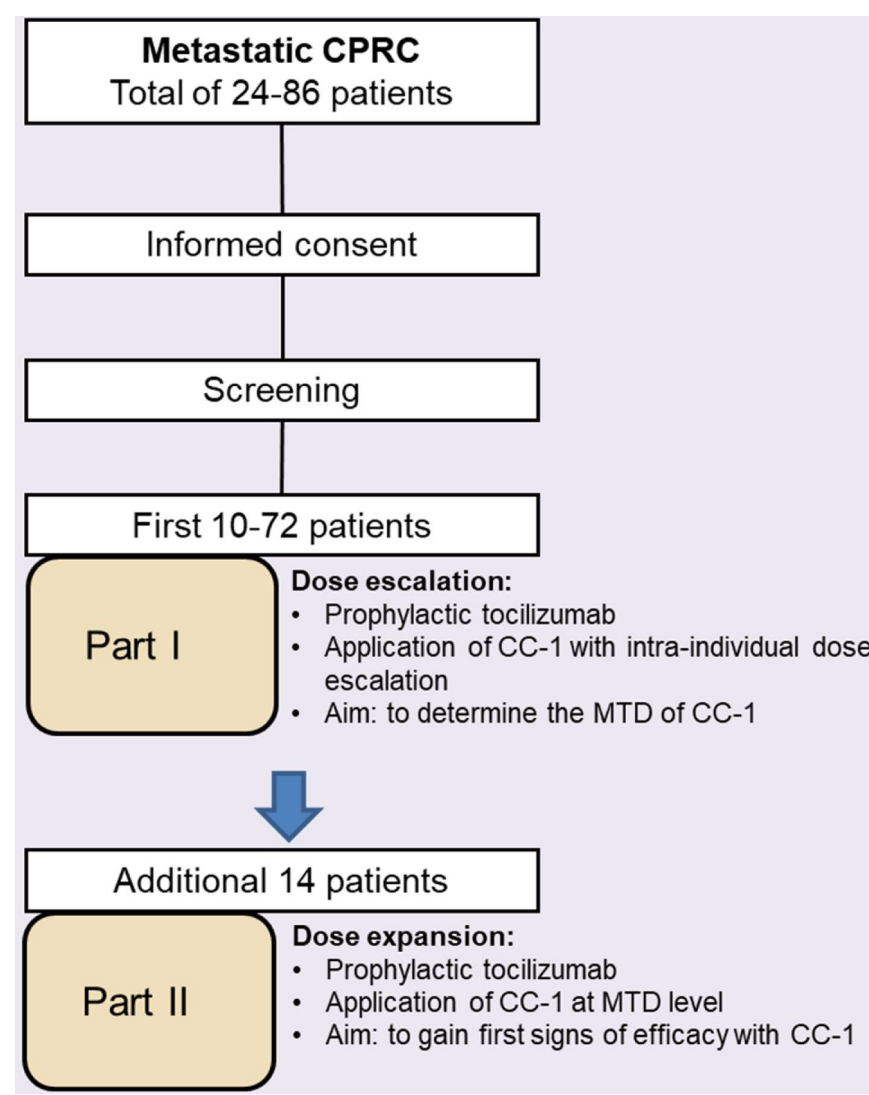

Figure 1 Study overview. CC-1, bispecific PSMAxCD3 antibody; CRPC, castration-resistant prostate carcinoma; MTD, maximum tolerated dose.

\section{Justification of starting and target dose}

Definition of the starting dose (28 $\mu \mathrm{g}$ corresponding to $0.14 \mathrm{nmol}$ ) was based on preclinical data obtained with CC-1 and considerations taking into account available results of clinical studies with similar constructs. In vitro, CC-1 induced relevant $\mathrm{T}$ cell activation at concentrations of approximately $10 \mathrm{ng} / \mathrm{mL}$. These data are provided in the investigators' brochure of the study and contained in a manuscript reporting the preclinical characterisation of CC-1 (Zekri et al, submitted). On application of the BiTE antibody blinatumomab, a $40 \mu \mathrm{g}$ dose reportedly leads to serum concentrations in humans of approximately $1 \mathrm{ng} /$ $\mathrm{mL}$ corresponding to $0.02 \mathrm{~nm}^{23}$ which results in increased cytokine levels. ${ }^{24}$ Due to the difference in molar weight between blinatumomab and CC-1, the molar dose of CC-1 is about four times lower than that of blinatumomab and thus four times lower molar serum concentrations will be achieved for CG-1 at identical 'mass dosing'. Considering the results of our preclinical analyses, this ensures a sufficient safety margin, in particular when treatment is started with $28 \mu \mathrm{g}$, that is, one dose level below $40 \mu \mathrm{g}$.

The target dose was determined based on in vivo dose titration studies in immunodeficient mice bearing large established tumours after adoptive transfer of human peripheral blood mononuclear cell. Based on dose titration experiments, it was concluded that application of approximately $1.2 \mathrm{mg}$ via continuous infusion per day
A
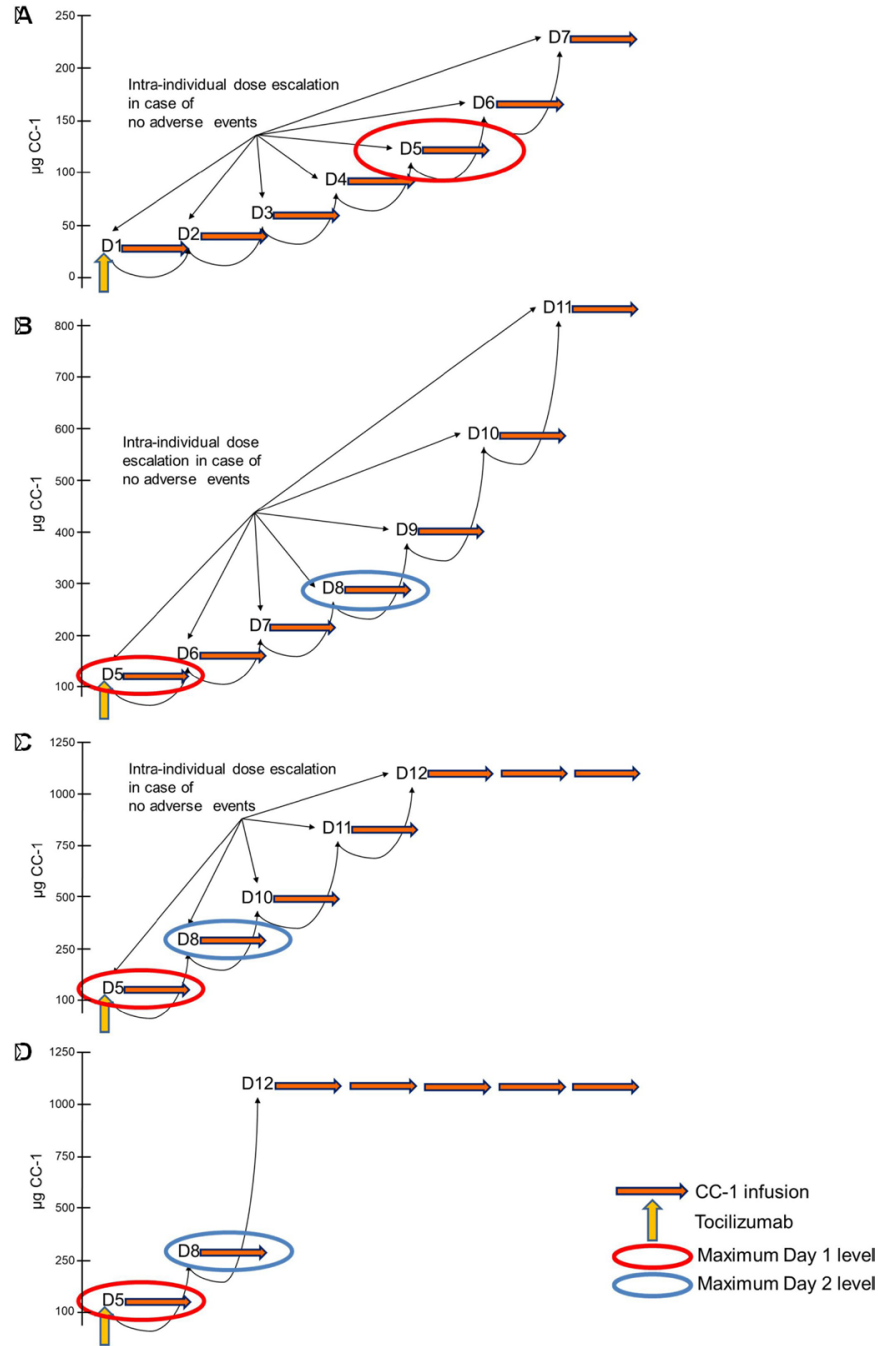

Figure 2 Dosing in different parts of CC-1 study. Part l: intraindividual dose escalation: tocilizumab $(8 \mathrm{mg} / \mathrm{kg}$ body weight) is applied once as pretreatment $>1$ hour prior to the start of CC-1 infusion on day 1 of each cycle. CC- 1 is administered as a 24-hour continuous intravenous infusion with escalated doses of CC-1. We perform a daily, intraindividual dose escalation to the next higher dose level. Dose escalations are depicted in the first patient (A), second (B), third (C). (D) Fourth to tenth patient of part I and part II: dose expansion at maximum tolerated dose (MTD): after preemptive interleukin-6 receptor blockade CC- 1 is administered as a 24-hour continuous intravenous infusion started at the MTD dose level. The dose levels applied on day 1 and day 2 must not exceed $110 \mu \mathrm{g}$ (maximum day 1 level) and $300 \mu \mathrm{g}$ (maximum day 2 level), respectively. CC-1, bispecific PSMAxCD3 antibody.

in humans would result in serum levels in the range of approximately $25-250 \mathrm{ng} / \mathrm{mL}$, which in mice was sufficient to eliminate established tumours. It is envisaged that dose levels within this range will be sufficient to achieve significant antitumour activity in humans; taking into consideration the step-dosing approach described below, $1156 \mu \mathrm{g}$ was chosen as maximum tested dose for the planned clinical study. 


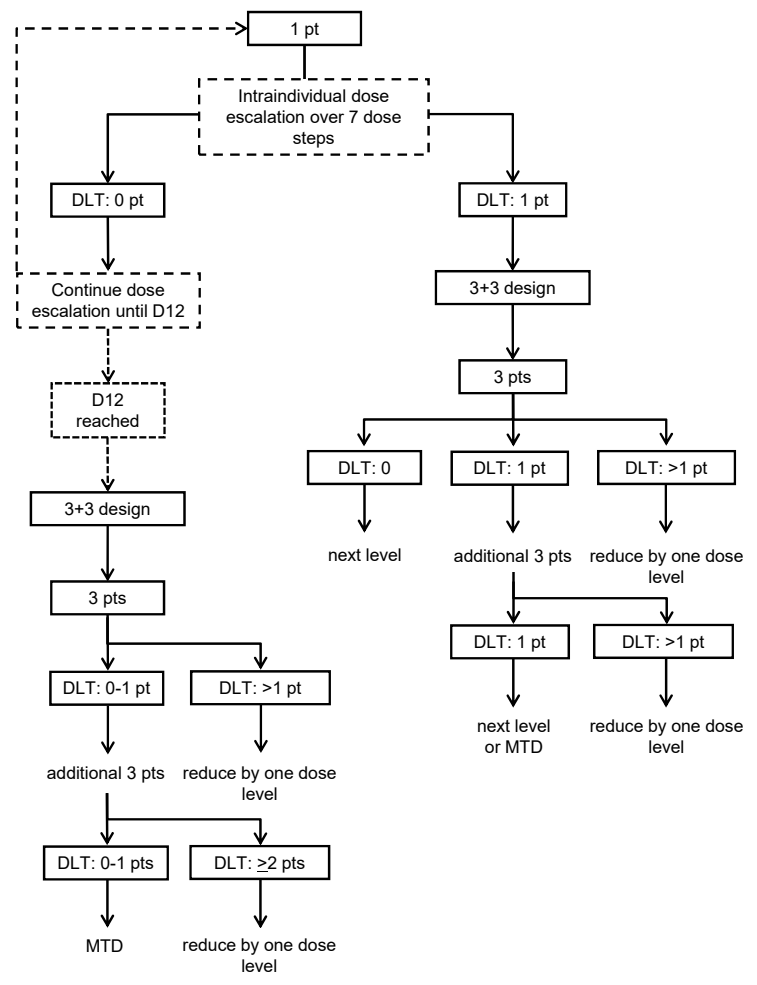

Figure 3 Flow chart of phase I design. In part of the phase I this is a dose escalation. Rather than the classical $3+3$ design our escalation is intraindividual based in case no doselimiting toxicity (DLT) occurs until maximum tolerated dose (MTD) is reached. If a DLT is reported, we will switch back to $3+3$ design. $\mathrm{D}$, dose level; pt, patient.

\section{Inclusion criteria}

Patients must meet the following criteria prior to treatment in the CC-1 study:

- Existence of a written informed consent.

- Patient is able to understand and comply with the protocol for the duration of the study including undergoing treatment and scheduled visits and examinations.

- CRPC after third-line therapy. Patients must be progressive prior to study enrolment, documented by either rising tumour marker or radiological assessment. Prior to study enrolment patients should have received chemotherapy and abiraterone or enzalutamide.

- Life expectance of $>3$ months.

- At least one measurable lesion that can be accurately assessed at baseline by CT or MRI and is suitable for repeated assessment.

- Eastern Cooperative Oncology Group Performance Status $\leq 2$.

- Patient aged $\geq 18$, no upper age limit.
- Male patients with partners of childbearing potential, who are sexually active, must agree to the use of two highly effective forms of contraception. This should be started from the signing of the informed consent and continue throughout period of taking study treatment for 3 months after last dose of study drug.

- Adequate bone marrow, renal and hepatic function defined by laboratory tests within 14 days prior to study treatment.

\section{Exclusion criteria}

Patients meeting any of the following criteria will not be considered for admission to the CC-1 study:

- Other malignancy within the last 5 years except: adequately treated non-melanoma skin cancer.

- Concurrent or previous treatment within 30 days in another interventional clinical trial with an investigational anticancer therapy.

- Persistent toxicity ( $\geq 2$ grade according to Common Terminology Criteria for Adverse Events (CTCAE) V.5.0) caused by previous cancer therapy, excluding alopecia and neurotoxicity ( $\leq 2$ grade).

- Clinical signs of active infection ( $>2$ grade according to CTCAE V.5.0).

- History of HIV infection.

- Immunocompromised patients.

- Active or chronic viral hepatitis (hepatitis B or hepatitis $\mathrm{C}$ virus).

- History of autoimmune disease.

- History of relevant central nervous systems (CNS) pathology or current relevant CNS pathology (eg, seizure, paresis, aphasia, cerebrovascular ischaemia/ haemorrhage, severe brain injuries, dementia, Parkinson's disease, cerebellar disease, organic brain syndrome, psychosis, coordination or movement disorder).

- Epilepsy requiring pharmacological treatment.

- Therapeutic anticoagulation therapy.

- Major surgery within 4 weeks of starting study treatment. Patients must have recovered from any effects of major surgery.

- Patients receiving any systemic chemotherapy or radiotherapy within 2 weeks prior to study treatment or a longer period depending on the defined characteristics of the agents used.

- Heart failure New York Heart Association III/IV.

- Severe obstructive or restrictive ventilation disorder.

- Known history of gastrointestinal perforation.

- Pre-existing human antihuman antibodies (HAHA).

- Known intolerance to CC-1, tocilizumab or other immunoglobulin drug products as well as hypersensitivity to any of the excipients present in the respective drug products (CC-1, tocilizumab).

\section{Treatment/study distribution}

As mentioned before, the study will be divided in two parts with similarly structured treatment cycles. Each cycle consists of 21 days, comprising 7 days (d1-7) of 


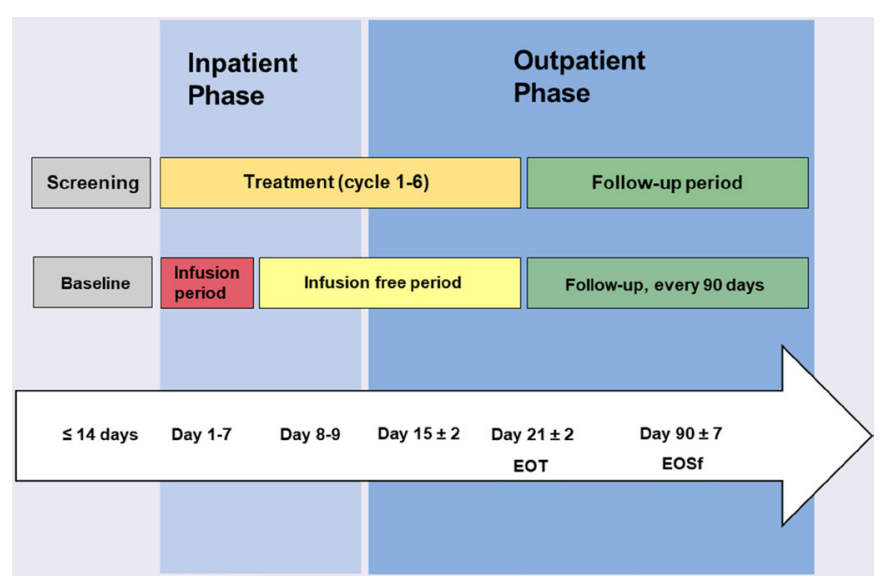

Figure 4 Study schedule. Screening: $\leq 14$ days. Assessment of baseline values for imaging and quality of life; infusion period: days 1-7 (hospitalised as inpatient); on day 1 baseline assessment for prostate-specific antigen, cytokines and liquid biopsy, vital signs, concomitant medication, signs and symptoms of disease; infusion free period: days 8-9 no infusion, but still hospitalised as inpatient (discharge on day 9); day 9/10-21 $( \pm 2)$ outpatient; visit at day $15( \pm 2)$ and 21 $( \pm 2)$ (end of treatment/EOT); follow-up period: outpatient, visit at day $90( \pm 7)$ (end of safety follow-up, EOSf); after EOSf follow-up every 90 days (up to one 1 year, ongoing adverse events have to be followed to resolution).

CC-1 application as continuous infusion, followed by a treatment free period with appended close monitoring for safety (inpatient and outpatient monitoring). The duration of the trial for each patient is expected to be at least 3 months including the first treatment cycle of 21 days (end of treatment) and a follow-up visit on day 90 (end of safety follow-up (EOSf)). Thereafter, a continuous survival follow-up every 3 months is conducted for 1 year after EOSf (figure 4). In case of clinical benefit, additional CC-1 cycles may be applied. The estimated duration of the clinical phase is 30 months.

\section{Dose escalation part}

Tocilizumab $(8 \mathrm{mg} / \mathrm{kg}$ body weight $)$ is applied once as pretreatment $>1$ hour prior to the start of CC- 1 infusion on day 1 of each cycle. CC-1 is administered as a 24-hour continuous intravenous infusion with escalated doses of CC-1 (absolute doses 28-1157 $\mu$ g, table 1). To rapidly achieve effective CC-1 doses, we perform a daily, intraindividual dose escalation to the next higher dose level (figure 2A).

If no dose-limiting toxicity (DLT) is observed, the achieved dose (on day 7) minus one dose level is the day 1 starting dose of the next patient. In general, the dose levels applied on day 1 and day 2 must not exceed $110 \mu \mathrm{g}$
(D5) and $300 \mu \mathrm{g}$ (D8), respectively (table 1). Those dose levels were implemented as safety steps with the calculated (higher) dose level to be shifted to day 2 or day 3 accordingly (compare figure 2B).

For example, if the first patient accomplishes D7, the next patient receives on day $1110 \mu \mathrm{g}$ (D5) and continues with further dose escalation; if this patient reaches D11 without DLT, the next patient receives on day 1 and day $2110 \mu \mathrm{g}$ (D5) and $300 \mu \mathrm{g}$ (D8), respectively before continuing with D10 and further escalation.

In case of observed DLT, the last tested dose according to schedule without observed DLT is applied to the next patient in a standard $3+3$ dose-escalating design without intraindividual dose escalation until MTD is defined.

After completion of the cycle of the previous patient and prior to dosing of the next patient (until maximum dose level D12=1157 $\mu \mathrm{g}$ ), approval by the Data Safety Monitoring Board (DSMB) is obtained. In case no DLT is observed, at least six patients are treated at the highest dose level before proceeding to the expansion cohort (figure 3).

\section{Dose expansion part}

After pre-emptive IL-6R blockade CC-1 is administered as a 24-hour continuous intravenous infusion started at the MTD dose level identified in the dose escalation part of the study. The dose levels applied on day 1 and day 2 must not exceed $110 \mu \mathrm{g}$ and $300 \mu \mathrm{g}$, respectively, which are implemented as safety steps with the MTD dose level shifted to day 2 or day 3 accordingly (figure 2B). This may result in application of MTD dose level for 5 (or 6) days in each cycle.

\section{Additional CC-1 treatment cycles}

We implemented the option for additional CC-1 treatment cycles in order that patients at an early stage of clinical development, in addition to receiving intraindividual dose escalation, may achieve maximum personal benefit. If patients are considered to benefit from study treatment (indicated for example, by a prostate-specific antigen (PSA) drop of $20 \%$ from baseline on day 15 to enable early decision making with regard to potential benefits in light of the required hospitalisation to avoid undue burden for the patient), additional cycles of CC-1 may be applied to the same patient with the exact same dose schedule as in the previous cycle. Each patient may receive up to six cycles in total.

\section{Endpoints of the study}

The primary endpoints of the study are incidence and severity of adverse events (AEs) (CTCAE V.5.0) over 21

Table 1 Dose levels (D) from starting dose to maximum tested dose

\begin{tabular}{lllllllllllll}
\hline Level & D1 & D2 & D3 & D4 & D5 & D6 & D7 & D8 & D9 & D10 & D11 & D12 \\
\hline Factor & - & $\sqrt{ } 2$ & $\sqrt{ } 2$ & $\sqrt{ } 2$ & $\sqrt{ } 2$ & $\sqrt{ } 2$ & $\sqrt{ } 2$ & $\sqrt{ } 2$ & $\sqrt{ } 2$ & $\sqrt{ } 2$ & $\sqrt{ } 2$ & $\sqrt{ } 2$ \\
CC-1 dose $(\mu \mathrm{g})$ & 28 & 40 & 56 & 78 & 110 & 153 & 215 & 300 & 422 & 590 & 826 & 1157 \\
\hline
\end{tabular}


days (ie, until end of first treatment cycle). Moreover, we report on DLT as important parameter to define MTD for the dose expansion part of the study. As part of a translational research programme, among others, the analysis of CC-1 half-life, the induced immune response as well as the molecular profiling in liquid biopsies, comprise the secondary endpoints and are:

- Safety (general): incidence and severity of AEs (CTCAE V.5.0).

- Immunogenicity: number and percentage of subjects who develop HAHA as compared with baseline.

- Cytokine induction: cytokine levels in serum.

- Pharmacokinetics: CC-1 serum concentrations.

- Antitumour activity: absolute changes from baseline in the tumour-marker (PSA) and objective tumour response assessed by response evaluation criteria in solid tumours (RECIST) on routine imaging.

- Survival: overall and progression free survival (absence of radiologically confirmed progression or death by any cause) evaluated by Kaplan-Meier method.

- Quality of life: overall quality of life scores (European Organization for Research and Treatment of Cancer core quality of life questionnaire).

\section{Safety/DLT}

Toxicity will be graded according to the National Cancer Institute CTCAE, V.5.0 and the CRS grading system by Lee $e t a l .{ }^{25}$ All AEs and serious AEs will be documented and reported accordingly to good clinical practice guidelines. Furthermore, we will report on adverse events of special interest (AESIs), which include occurrence of allergic reaction, development of HAHA and CRS. These AESIs will be monitored closely and included in patient safety narrative reports to the DSMB.

DLT is defined as any grade $\geq 3 \mathrm{AE}$ that the investigator considers to be at least possibly related to study treatment and occurring within the DLT safety period (from day 1 to 21) with few exceptions. In addition, since tocilizumab is given pre-emptively, the safety profile of tocilizumab is also considered. Remarkably, AEs occurring in the context of CRS are only considered as DLT, if the CRS grading is $\geq 3$ according to Lee $e t a .^{25}$

\section{Data Safety Monitoring Board}

In the dose escalation part of the study, the DSMB receives patient safety narrative reports routinely after each patient in which dose has been escalated (assessment on day 21). After completion of the cycle of the previous patient, approval by the DSMB must be obtained prior to dosing of the next patient (until maximum dose level is reached). At maximum dose level of CC-1, the DSMB will receive a report routinely after the first three and second three (this is prior to start of the expansion part) treated patients. In addition, safety narrative reports are created on occurrence of DLT.

\section{Sample size calculation}

Depending on the occurrence of DLT in the dose escalation part, the patient number to define the MTD varies. A minimum number of 24 patients are treated, but potentially up to 86 patients may be included into the trial. Based on the broad range of dose levels to be tested (absolute doses of 28-1157 $\mu \mathrm{g}$ ) and the employed intraindividual dose escalation (figure 2A), sample size may vary considerably dependent on CC-1-induced AEs. The dose escalation calculation is rule based on the titration design by Simon et al. ${ }^{26}$ Once the maximum tested dose is reached, additional six patients are treated to define MTD (figure 3). Thus, the sample size varies in the dose escalation part of the study between $n=10$ patients required for definition of the MTD in the minimal and $\mathrm{n}=72$ patients in the maximum case. Subsequently, a dose expansion part with 14 patients is foreseen to gain more information about the MTD level and to better define the recommended phase II dose of CC-1. Based on the planning, in total 20 patients will be treated at the MTD level, 6 patients from the dose escalation phase and 14 patients of the expansion cohort. Thus, we will be able to estimate within a single-stage phase II design an objective response defined as PSA drop $\geq 50 \%$ estimating P0 the maximum response proportion of a poor drug of $\leq 30 \%$ of the patients, and $\mathrm{P} 1$ the minimum response proportion of a good drug of $\geq 60 \%$ with a power of $80 \%$ and a type one error of $5 \%$. To this aim we will need $n=17$ evaluable patients assuming a drop-out rate of $15 \%(n=3)$.

Estimation of sample size:

Minimum: ( 4 escalation +6 highest dose $)+14$ expansioncohort $=24$ patients

Maximum: $12 \times 6 \quad$ escalation-cohort +14 expansioncohort $=86$ patients

\section{Patient and public involvement}

CC-1 production (in accordance to good manufacturing practice) and trial conduct are exclusively funded by public resources without contribution of pharmaceutical industry or commercial organisations. Prior to approval of funding, proposals were peer reviewed in a competitive manner by the Helmholtz Validation Fund and the Joint Funding Program of the German Cancer Consortium.

Design of the clinical trial was done without support by patient representatives, but extensive efforts were undertaken to inform patients about the beginning of study recruitment.

\section{Data handling and storage}

All findings including clinical, radiological and laboratory data will be documented by the investigator or an authorised member of the study team in the electronic case report form. Investigators guarantee the privacy of patients and personal data are treated according to the European general data protection regulation (EU 2016/679) and the German law. The data will be stored for 25 years. All data entry, modification or deletion, will be recorded automatically in an electronic trail. 
Monitoring of data will be conducted on a regular basis and prior to each safety report for DSMB.

\section{ETHICS AND DISSEMINATION}

The study will be performed in accordance with the Declaration of Helsinki and will comply to the International Conference on Harmonization and Good Clinical Practice. All possible treatments and examinations for CRPC are undertaken after obtaining written informed consent from (see online supplemental file) the patients by the treating physician. This trial is funded by research grants from the Helmholtz Validation Fund and the Joint Funding Program of the DKTK. The CC-1 study was approved by the Ethics Committee of the University and University Hospital Tübingen (100/2019AMG1) and the federal institute for biomedicine and vaccine, the Paul-Ehrlich-Institute, Germany (3684/02). During trial conduct, the responsible authorities will be informed on a regular basis about the progress of the trial. The results of this clinical trial results will be presented at relevant national and international meetings and published in peer-reviewed journals regardless of outcome. All planned publications will be reviewed by the principal investigator and the biostatistician prior to publication to avoid violation of patients' rights.

\section{DISCUSSION}

As of now, metastatic CRPC is an often rapidly progressive disease without curative treatment options. To address this high medical need, we here introduce a FIH study evaluating safety and efficacy of the bispecific PSMAxCD3 antibody CC-1 in patients with CRPC after failure of thirdline therapy.

CC-1 is developed in a novel IgG-like format termed IgGsc to overcome several problems of so far available bsAb constructs: drawbacks in particular of bsAbs in the BiTE format are their low serum half-life (approximately 1 hour) ${ }^{27}$ and aggregation tendency, ${ }^{28}$ which necessitates cumbersome application protocols and results in offtarget activation of $\mathrm{T}$ cells, respectively. Besides blinatumomab, this also holds true for the BiTE PSMAxCD3 bsAb developed by Amgen that presently is undergoing evaluation in phase I studies. ${ }^{29}$ The IgGsc format of CC-1 not only allows for a longer serum half-life, but also has a lower aggregation tendency compared with the prototypical BiTe format, with accordingly reduced off-target T cell activation and thus fewer side effects ${ }^{27}{ }^{30} 31$ (Zekri $e t$ $a l$, submitted). In addition, to achieve our superordinate goal, the safe application of sufficiently high bsAb doses which in turn shall facilitate better clinical efficacy, we will employ pre-emptive IL-6R blockade to prevent rather than to treat CRS. The prophylactic application of tocilizumab should abolish the clinical effects of CRS without impairing $\mathrm{T}$ cell antitumour reactivity. ${ }^{32} 33$

An additional advantage of CC- 1 is that its target antigen PSMA is expressed on prostate carcinoma cells as well as on the tumour vessels of CRPC. Thereby, a dual mode of anticancer action is enabled: targeting the tumour vessels should allow for improved influx of $\mathrm{T}$ cells into the tumour via the damaged endothelial barrier followed by effective combating of the tumour cells themselves. Thereby we hope to overcome a critical factor that so far limits the success not only of bsAbs, but of $\mathrm{T}$ cell-based treatment of solid tumours in general. ${ }^{1819}$

Taking into account the lack of effective treatment options and the dismal prognosis in the study patient population, the expected benefits of a CC- 1 treatment with pre-emptive tocilizumab application in this clinical study outweigh the potential risks for the patients, especially since multiple risk mitigation measures have been implemented. The progress and safety data will be monitored by three independent experts (DSMB) and they will give approval/recommendations to the coordinating investigator/the sponsor whether to stop the trial or to change the trial protocol. In addition, the implemented intraindividual escalation of the applied CC-1 dose constitutes an additional hallmark for patients in this trial. Usually, early clinical trials study safety and tolerability of new drugs, with therapeutic benefit for patients accordingly being only of secondary interest. In our view, this constitutes an ethical dilemma, which we tried to resolve by our approach to rapidly increase CC-1 dose levels and thus reach doses levels that were preclinically effective already for the first patients treated. The concomitant prophylactic application of tocilizumab further supports the feasibility of the fast, intra-individual dose escalation and in turn the rapid evaluation of the MTD of CC-1 as first step. In case that no DLT is observed, already the fourth patient will receive the target dose. After six further patients that receive the maximum test dose, the dose escalation part would be completed, which constitutes an important particularity of our trial. The dose escalation phase is then followed by a dose expansion phase (also with prophylactic IL-6R blockade), as this approach has been shown to be efficient and beneficial for patients in early clinical trials. ${ }^{34}$ Furthermore, in the expansion phase we plan to implement PSMA-positron emission tomography analysis to obtain reliable information on target antigen expression in patients undergoing analysis for efficacy, as reduction of PSMA expression on malignant cells reportedly occurs in a substantial proportion of patients over time. ${ }^{35}$ Of note, even a few hundred molecules per cell suffice to render the malignant cells susceptible to $\mathrm{T}$ cell killing on bsAb treatment.

Notably, the development of CC-1 and the conduct of this trial are fully funded by public resources. This corresponds with our conviction that drug development, which nowadays almost exclusively is conducted by the pharmaceutical industry, should at least in part be reclaimed by academia as recently described elsewhere. ${ }^{36}$ 


\section{Author affiliations}

${ }^{1}$ Clinical Collaboration Unit Translational Immunology, German Cancer Consortium (DKTK), Department of Internal Medicine, University Hospital Tübingen, Tübingen, Germany

${ }^{2}$ Cluster of Excellence iFIT (EXC2180) 'Image-Guided and Functionally Instructed Tumor Therapies', University of Tübingen, Tübingen, Germany

${ }^{3}$ Institute for Cell Biology, Department of Immunology, University of Tübingen, Tübingen, Germany

${ }^{4}$ National Center of Tumor Diseases-Trial Center, National Center of Tumor Diseases, German Cancer Research Center, Heidelberg, Germany

${ }^{5}$ Department of Internal Medicine VI, Heidelberg University Hospital, Heidelberg, Germany

${ }^{6}$ Department of Internal Medicine V, Heidelberg University Hospital, Heidelberg, Germany

Contributors JSH, JSW, GJ, RFS and HRS designed the study protocol. MP and JK supervised production of CC-1 according to GMP and provided preclinical data to be provided to regulatory authorities. JSH, JSW, HRS and RFS initiated and conducted the clinical study. JSH, HRS and RFS wrote the paper. JSW, GJ, MP and JK contributed to manuscript preparation. All authors read, revised and approved the submitted manuscript.

Funding This trial is funded by research grants from the Helmholtz Validation Fund (HVF0055/OPTIMAB) and the Joint Funding Program of the German Cancer Consortium (DKTK). Sponsor of the study is the University Hospital Tübingen.

Competing interests GJ and HRS are listed as inventors on the patent application 'Novel PSMA binding antibody and uses thereof', EP16151281 and others, with the German Cancer Research Center (DKFZ), Heidelberg, Germany, as applicant. The other authors declare no competing interests.

Patient consent for publication Not required.

Ethics approval The CC-1 study (protocol version 1.2, dated 12.08.2019) was approved by the Ethics Committee of the University and University Hospital Tübingen (100/2019AMG1) and the federal institute for biomedicine and vaccine, the Paul-Ehrlich-Institute, Germany (3684/02).

Provenance and peer review Not commissioned; externally peer reviewed.

Open access This is an open access article distributed in accordance with the Creative Commons Attribution Non Commercial (CC BY-NC 4.0) license, which permits others to distribute, remix, adapt, build upon this work non-commercially, and license their derivative works on different terms, provided the original work is properly cited, appropriate credit is given, any changes made indicated, and the use is non-commercial. See: http://creativecommons.org/licenses/by-nc/4.0/.

ORCID iD

Jonas S Heitmann http://orcid.org/0000-0002-7305-8620

\section{REFERENCES}

1 WHO. World cancer report 2014. World Health Organisation, 2014.

2 Ferlay J, Soerjomataram I, Dikshit R, et al. Cancer incidence and mortality worldwide: sources, methods and major patterns in GLOBOCAN 2012. Int J Cancer 2015;136:E359-86.

3 Fizazi K, Scher HI, Molina A, et al. Abiraterone acetate for treatment of metastatic castration-resistant prostate cancer: final overall survival analysis of the COU-AA-301 randomised, double-blind placebo-controlled phase 3 study. Lancet Oncol 2012;13:983-92.

4 Ryan CJ, Smith MR, Fizazi K, et al. Abiraterone acetate plus prednisone versus placebo plus prednisone in chemotherapy-naive men with metastatic castration-resistant prostate cancer (COUAA-302): final overall survival analysis of a randomised, double-blind, placebo-controlled phase 3 study. Lancet Oncol 2015;16:152-60.

5 Scher HI, Fizazi K, Saad F, et al. Increased survival with enzalutamide in prostate cancer after chemotherapy. N Engl J Med 2012;367:1187-97.

6 Berthold DR, Pond GR, Soban F, et al. Docetaxel plus prednisone or mitoxantrone plus prednisone for advanced prostate cancer: updated survival in the Tax 327 study. J Clin Oncol 2008;26:242-5.

7 de Bono JS, Oudard S, Ozguroglu M, et al. Prednisone plus cabazitaxel or mitoxantrone for metastatic castration-resistant prostate cancer progressing after docetaxel treatment: a randomised open-label trial. Lancet 2010;376:1147-54.

8 Ahmadzadehfar H, Rahbar K, Kürpig S, et al. Early side effects and first results of radioligand therapy with 177Lu-DKFZ-617 PSMA of castrate-resistant metastatic prostate cancer: a two-centre study. EJNMMI Res 2015:5:114.

9 Weber J, Mandala M, Del Vecchio M, et al. Adjuvant nivolumab versus ipilimumab in resected stage III or IV melanoma. N Engl J Med 2017;377:1824-35.

10 Motzer RJ, Tannir NM, McDermott DF, et al. Nivolumab plus ipilimumab versus sunitinib in advanced renal-cell carcinoma. $N$ Engl J Med 2018:378:1277-90.

11 Paz-Ares L, Luft A, Vicente D, et al. Pembrolizumab plus chemotherapy for squamous non-small-cell lung cancer. $N$ Engl $J$ Med 2018;379:2040-51.

12 Fay AP, Antonarakis ES. Blocking the PD-1/PD-L1 axis in advanced prostate cancer: are we moving in the right direction? Ann Trans/ Med 2019;7:S7.

13 Barrett DM, Grupp SA, June CH. Chimeric antigen receptor- and TCR-Modified T cells enter main street and wall Street. J Immunol 2015; $195: 755-61$

14 Topp MS, Gökbuget N, Stein AS, et al. Safety and activity of blinatumomab for adult patients with relapsed or refractory Bprecursor acute lymphoblastic leukaemia: a multicentre, single-arm, phase 2 study. Lancet Oncol 2015;16:57-66.

15 Topp MS, Gökbuget N, Zugmaier G, et al. Phase II trial of the anti-CD19 bispecific $T$ cell-engager blinatumomab shows hematologic and molecular remissions in patients with relapsed or refractory B-precursor acute lymphoblastic leukemia. J Clin Oncol 2014;32:4134-40.

16 Pang $\mathrm{Y}$, Hou X, Yang C, et al. Advances on chimeric antigen receptormodified T-cell therapy for oncotherapy. Mol Cancer 2018;17:91.

17 Hoseini SS, Dobrenkov K, Pankov D, et al. Bispecific antibody does not induce T-cell death mediated by chimeric antigen receptor against disialoganglioside GD2. Oncoimmunology 2017;6:e1320625.

18 Sondel PM, Sosman JA, Hank JA, et al. Tumor-infiltrating lymphocytes and interleukin-2 in melanomas. N Engl J Med 1989;320:1418-9.

19 Ganss R, Ryschich E, Klar E, et al. Combination of T-cell therapy and trigger of inflammation induces remodeling of the vasculature and tumor eradication. Cancer Res 2002;62:1462-70.

20 Le RQ, Li L, Yuan W, et al. FDA approval summary: tocilizumab for treatment of chimeric antigen receptor $T$ cell-induced severe or lifethreatening cytokine release syndrome. Oncologist 2018;23:943-7.

21 Qian JH, Titus JA, Andrew SM, et al. Human peripheral blood lymphocytes targeted with bispecific antibodies release cytokines that are essential for inhibiting tumor growth. $J$ Immunol 1991;146:3250-6.

22 Jung G, Salih H. Novel PMSA binding antibody and uses thereof, 2017.

23 Klinger M, Brandl C, Zugmaier G, et al. Immunopharmacologic response of patients with $B$-lineage acute lymphoblastic leukemia to continuous infusion of T cell-engaging CD19/CD3-bispecific BiTE antibody blinatumomab. Blood 2012;119:6226-33.

24 Topp MS, Kufer P, Gökbuget N, et al. Targeted therapy with the T-cell-engaging antibody blinatumomab of chemotherapy-refractory minimal residual disease in B-lineage acute lymphoblastic leukemia patients results in high response rate and prolonged leukemia-free survival. J Clin Oncol 2011;29:2493-8.

25 Lee DW, Gardner R, Porter DL, et al. Current concepts in the diagnosis and management of cytokine release syndrome. Blood 2014;124:188-95.

26 Simon R, Freidlin B, Rubinstein L, et al. Accelerated titration designs for phase I clinical trials in oncology. $J$ Nat/ Cancer Inst 1997;89:1138-47.

27 Durben M, Schmiedel D, Hofmann M, et al. Characterization of a bispecific FLT3 X CD3 antibody in an improved, recombinant format for the treatment of leukemia. Mol Ther 2015;23:648-55.

28 Wörn A, Plückthun A. Stability engineering of antibody single-chain Fv fragments. J Mol Biol 2001;305:989-1010.

29 Klinger M, Benjamin J, Kischel R, et al. Harnessing T cells to fight cancer with BiTE $\AA$ antibody constructs--past developments and future directions. Immunol Rev 2016;270:193-208.

30 Jung G, Honsik CJ, Reisfeld RA, et al. Activation of human peripheral blood mononuclear cells by anti-T3: killing of tumor target cells coated with anti-target-anti-T3 conjugates. Proc Natl Acad Sci U S A 1986;83:4479-83.

31 Pflugler M, Vogt F, Zekri L, et al. CC-1, a bispecific PSMA antibody in an optimized format for treatment of prostate cancer and squamous cell carcinoma of the lung. Oncol Res Treat 2018;41:234-34.

32 Davila ML, Riviere I, Wang X, et al. Efficacy and toxicity management of 19-28z CAR T cell therapy in B cell acute lymphoblastic leukemia. Sci Transl Med 2014;6:224ra25.

33 Teachey DT, Rheingold SR, Maude SL, et al. Cytokine release syndrome after blinatumomab treatment related to abnormal 
macrophage activation and ameliorated with cytokine-directed therapy. Blood 2013;121:5154-7.

34 Chakiba C, Grellety T, Bellera C, et al. Encouraging trends in modern phase 1 oncology trials. N Engl J Med 2018;378:2242-3.

35 Mannweiler S, Amersdorfer P, Trajanoski S, et al. Heterogeneity of prostate-specific membrane antigen (PSMA) expression in prostate carcinoma with distant metastasis. Pathol Oncol Res 2009;15:167-72.

36 Salih HR, Jung G. The challenges of translation. EMBO Mol Med 2019;11:e10874. 